\title{
Ist Cannabidiol ein Lebensmittel oder ein Arzneimittel?
}

\author{
Eine Positionierung
}

\author{
Karin Kraft ${ }^{1}$, Mathias Schmidt ${ }^{2}$ \\ 1 Lehrstuhl für Naturheilkunde, Zentrum für Innere Medizin, Universitätsmedizin Rostock \\ 2 Herbresearch Germany, Mattsies
}

\section{ZUSAMMENFASSUNG}

CBD-haltige Produkte wie sogenannte CBD-Öle werden in Form von Nahrungsmitteln und Nahrungsergänzungsmitteln vertrieben und vielfach mit medizinischen Indikationen bei schweren Erkrankungen beworben. Derzeit werden auf europäischer Ebene Anträge auf Novel-Food-Status bearbeitet. Bei genauerer Betrachtung erweisen sich jedoch bereits die Grundlagen für die Einstufung von CBD als Lebensmittel als fragwürdig. CBD ist eindeutig ein verschreibungspflichtiger Arzneistoff mit bekannten pharmakologischen Effekten, der im Falle von Cannabisextrakten zudem Betäubungsmittelstatus hat. Für die Verwendung in Nahrungsmitteln fehlen die lebensmitteltypischen Eigenschaften. Auch wenn CBD selbst in hohen Dosen toxikologisch unbedenklich ist: Durch eine nachgewiesene Anwendungssicherheit wird ein Arzneistoff nicht zum Lebensmittel.

\section{Einleitung}

Die neuen gesetzlichen Grundlagen vom 10. März 2017 für den medizinischen Einsatz von Cannabiszubereitungen haben zu einer Vielzahl von Produkten auf dem Markt und zu erheblicher Verunsicherung hinsichtlich des regulatorischen Status solcher Produkte geführt. Dies gilt vor allem für Lebensmittel, welche Cannabiszubereitungen enthalten, insbesondere solche mit dem Inhaltsstoff Cannabidiol (CBD). Aufgrund eines EuGH-Urteils vom 19. November 2020 kam die EU-Kommission am 3.12.2020 zu der Schlussfolgerung, dass Cannabidiol-Produkte prinzipiell als Lebensmittel eingestuft werden können (https://ec.europa.eu/germany/news/

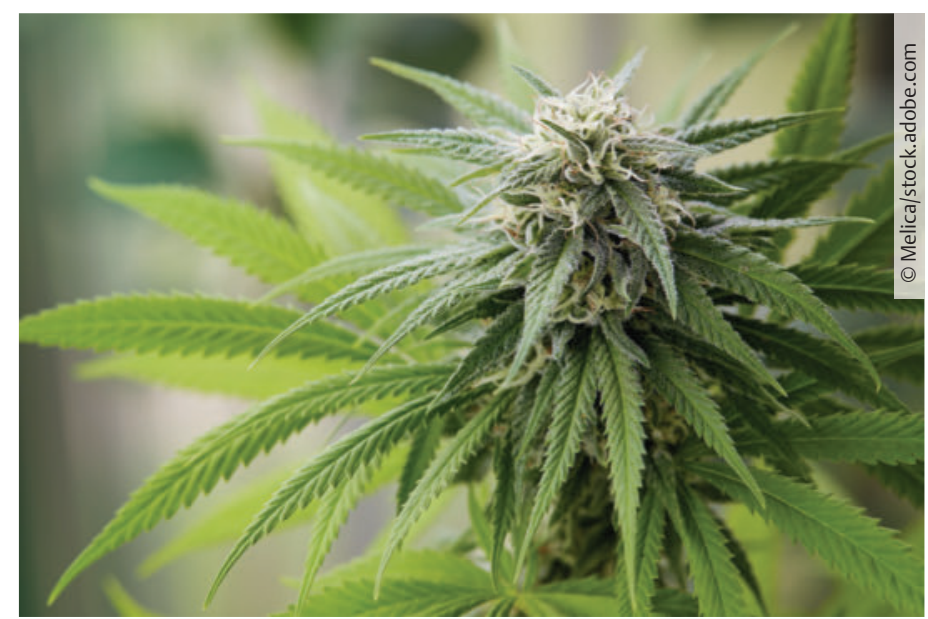

Abb. 1 Cannabis sativa L. (C) Melica/stock.adobe.com 20201203-cannabidiol-produkte_de). Diese Aussage wird aber offenbar nur aus der höchstgerichtlichen Aussage abgeleitet, dass CBD kein Suchtstoff ist. Kann daraus wirklich geschlossen werden, der EuGH unterstütze damit den Einsatz von CBD in Lebensmitteln?

Im Folgenden soll zunächst der aktuelle Stand der Abgrenzungsfragen angesprochen werden, bevor daraus Empfehlungen abgeleitet werden.

\section{Hintergründe}

Cannabis sativa (Hanf) ist eine Pflanzenart, die aus vielen Sorten besteht, die industriell (Nutzhanf) oder medizinisch (Medizinalhanf) genutzt werden ( $\triangleright$ Abb. 1).

Von der EU wurden 52 Hanfsorten, die weniger als 0,2\% THC enthalten, für den Nutzanbau zertifiziert. Hanffasern dienen als Rohstoff für die Textilindustrie, die Hanfsamen und das aus ihnen gepresste Hanföl werden als hochwertige Lebensmittel genutzt, z. B. als Hanfmehl, Trinkhanf oder Hanfbutter. Sorten von Cannabis indica Lam. und Cannabis sativa L. werden als Medizinalhanf verwendet.

Die weiblichen Blüten von Cannabis sativa L. produzieren und sezernieren in speziellen Drüsenhaaren Cannabinoide, deren bekanntester Vertreter das psychoaktiv wirkende (-)- $\Delta^{9}$-trans-Tetrahydrocannabinol (THC) ist (> A bb. 2).

THC ist u.a. in dem ätherischen Haschischöl enthalten, das durch die Extraktion der Blütenstände mit unpolaren Lösungsmitteln erhalten wird. THC ist der Grund für die 
Verwendung von Cannabisblüten als Rauschdroge und die entsprechende Einstufung als Betäubungsmittel (BtM). Für den missbräuchlichen oralen Konsum werden Cannabis oder Haschisch mit stark fetthaltigen Nahrungsmitteln oder Trägersubstanzen verarbeitet. Diese Zubereitungsformen können direkt oder als Cannabisbutter in Gebäck verbacken oder mit Kakao oder Schokolade gebunden werden.

Ein weiterer wichtiger Vertreter der Phytocannabinoide ist das Cannabidiol (CBD), das keine psychotropen Effekte aufweist ( $\triangleright$ Abb. 3 ).

Zur Orientierung: In Cannabisproben aus Ungarn wurden 0,3-0,9\% CBD in Blättern und Blüten nachgewiesen [19] - eine entsprechende Menge sollte daher auch in Cannabisextrakten zu erwarten sein. Für CBD sind weder Abhängigkeit noch Missbrauch bekannt, weshalb die WHO die Einstufung von CBD als Suchtstoff ablehnt. CBD erfüllt nicht die Vorbedingungen für eine Klassifizierung unter das Betäubungsmittelrecht [12]. Neben CBD sind noch ca. 80 weitere Phytocannabinoide, wie z.B. Cannabinol, Cannabigerol oder Cannabichromen, mit unterschiedlichen Wirkungen bekannt.

\section{Hanfprodukte des Marktes}

Hanfsamen und daraus abgeleitete Lebensmittel wie Hanföl oder Hanfmehl gelten uneingeschränkt als Lebensmittel. Hanfsamen haben keine Drüsenhaare und enthalten daher keine Cannabinoide-eventuell bei der Analytik gefundenes THC stammt aus Kontaminationen der Samen bei der Ernte durch Kontakt mit den Blütenständen [7].

In den letzten Jahren kamen sehr viele Produkte mit Cannabiszubereitungen in den Handel, viele davon als Nahrungsmittel oder Nahrungsergänzungsmittel, aber auch Kosmetika und Tabakersatzprodukte mit angereicherten Cannabisextrakten oder isoliertem / synthetischem CBD eroberten den Markt [12]. Tabakersatz und kosmetische Produkte sind nicht Gegenstand dieser Ausarbeitung, sie werden aber in aktuellen Stellungnahmen thematisiert [20].

Im Folgenden soll die Fragestellung diskutiert werden, ob CBD ein Nahrungsmittel, Nahrungsergänzungsmittel, ein Lebensmittelzusatzstoff, ein Genussmittel, Novel Food oder Arzneimittel - evtl. mit BtM-Status - ist.

\section{DISCLAIMER}

Die folgenden Ausführungen sind nicht als Rechtsgutachten zu verstehen, sie spiegeln die Interpretation der Autoren wider.

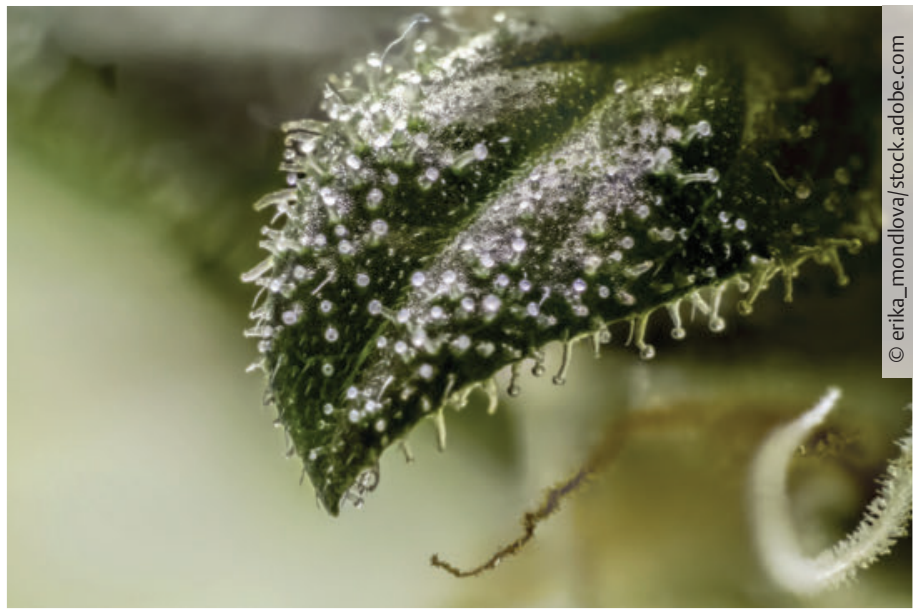

Abb. 2 Cannabinoide werden von der Pflanze in Drüsenhaare sezerniert. (c) erika_mondlova / stock.adobe.com

\section{Ist CBD ein Nahrungsmittel?}

Nach der Definition der EU-Lebensmittelverordnung (178/2002/ EC) sind Lebensmittel pflanzliche oder tierische Stoffe, die für die menschliche Ernährung geeignet sind. Zu den Lebensmitteln zählen Nahrungsmittel, Nahrungsergänzungsmittel, Genussmittel und Lebensmittelzusatzstoffe. Nahrungsmittel sind in der engeren Definition keine vollwertigen Lebensmittel, der Begriff wird in der Praxis aber äquivalent benutzt. Nahrungsmittel liefern Grundnährstoffe wie Proteine, Fette und Kohlenhydrate, aber auch Ballaststoffe, Vitamine, Mineralstoffe, Spurenelemente und Wasser. Diskutiert wird seit vielen Jahren auch die Bedeutung der sekundären Pflanzeninhaltsstoffe für die gesunde Ernährung. Eine Basisnährstofffunktion von CBD ist weder bekannt noch gegenwärtig erkennbar.

CBD-Öle werden nicht nur als Nahrungsergänzungsmittel (s. u. ), sondern auch als reguläre Nahrungsmittel angeboten, wobei jedoch ein hoher CBD-Gehalt nur durch artifiziellen Zusatz von CBD in Form von Konzentraten, Isolaten oder synthetisch gewonnenem CBD erreicht werden kann. Ein solcher Zusatz könnte regulatorisch unter die Lebensmittelzusatzstoffverordnung fallen. Der

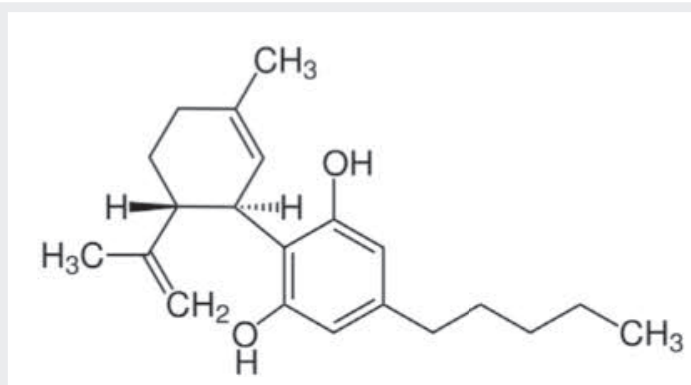

-Abb. 3 Strukturformel von Cannabidiol (CBD). 


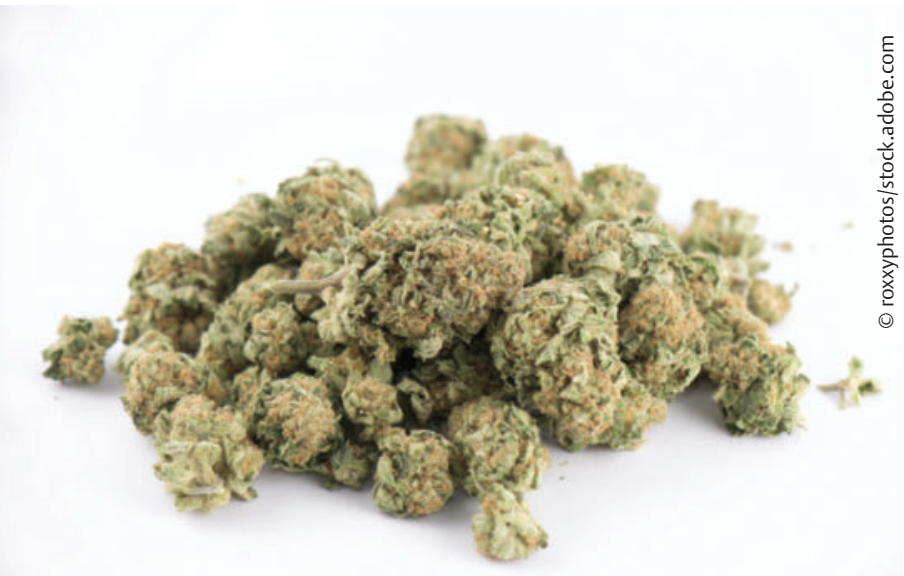

-Abb. 4 Handelsübliche Form der Cannabisblüten. @ roxxyphotos / stock.adobe.com

Vertrieb als natürliches Lebensmittel grenzt damit an Irreführung - zumindest aber sind solche Zubereitungen im Licht der Novel-Food-Regelungen zu betrachten.

\section{Schlussfolgerung zur Lebensmittelfunktion von CBD}

CBD-Öle oder andere Lebensmittel mit angereichertem CBD erfüllen keine der Basisfunktionen eines Lebensmittels.

\section{Ist CBD ein}

\section{Nahrungsergänzungsmittel?}

Die Nahrungsergänzungsmittelrichtlinie 2002/46/EC definiert in Artikel 2 Nahrungsergänzungsmittel als Einfach- oder Mehrfachkonzentrate von Nährstoffen oder sonstigen Stoffen mit ernährungsspezifischer oder physiologischer Wirkung. Im Detail werden als Nährstoffe nur Vitamine und Mineralstoffe genannt, nicht aber sekundäre Pflanzeninhaltsstoffe. Um pflanzliche Zubereitungen oder sekundäre Pflanzeninhaltsstoffe in Nahrungsergänzungsmitteln zu rechtfertigen, müssen daher deren ernährungsspezifische oder -physiologische Effekte aufgezeigt werden.

Sekundäre Pflanzeninhaltsstoffe sind regulärer und normaler Bestandteil der menschlichen Ernährung. Insbesondere für Polyphenole werden bereits seit längerer Zeit ernährungsphysiologische Effekte im Sinne einer vitaminähnlichen Funktion diskutiert [16],[22]. Ernährungsbedingte Basisfunktionen der in Cannabisblüten enthaltenen Polyphenole, zu denen u. a. auch das CBD gehört, sind nicht plausibel herleitbar, da Cannabisblüten bzw. CBD kein regulärer und normaler Bestandteil der menschlichen Ernährung sind ( $\mathbf{A} \mathbf{b b} \mathbf{4}$ ). Eine Situation, in welcher durch die übliche Ernährung nennenswerte Mengen an CBD zugeführt werden, ist kaum vorstellbar.

\section{Schlussfolgerung zur Einstufung als Nahrungsergänzungsmittel}

Eine ernährungsphysiologische Funktion von CBD und damit die Grundvoraussetzung der Definition eines Nahrungsergänzungsmittels gemäß der Nahrungsergänzungsmittelrichtlinie 2002/46 / EC ist nicht gegeben.

\section{Kann CBD ein Genussmittel sein?}

Der Begriff des Genussmittels ist nicht klar definiert. Die übliche Definition umfasst Lebensmittel, die nicht wegen ihres Nährwerts, sondern wegen ihres Geschmacks und/oder ihrer anregenden Wirkung aufgenommen werden, z. B. Kaffee, Tee oder alkoholhaltige Getränke. CBD hat dagegen keine anregende oder dämpfende Wirkung.

Schlussfolgerung zur Einstufung als Genussmittel CBD wirkt weder dämpfend noch anregend. Für eine Klassifizierung von CBD als Genussmittel fehlen die Voraussetzungen.

\section{Kann CBD ein Lebensmittelzusatzstoff sein?}

Lebensmittelzusatzstoffe werden durch die Verordnung 1333/2008/EC geregelt. Es sind Substanzen, welche „die Eigenschaften von Lebensmitteln verbessern, ihren Geschmack beeinflussen, das Aussehen, die Haltbarkeit verbessern oder die technologische Verarbeitung erleichtern“ (https://www.bvl.bund.de/D E/Arbeitsbereiche/01_Lebensmittel/03_Verbraucher/05 _Zusatzstoffe/lm_zusatzst_node.html). Die gesetzliche Grundlage umfasst klar definierte Kategorien von Zusatzstoffen. Diese sogenannten Funktionsklassen sind Anhang I der Verordnung 1333/2008/EC zu entnehmen ( $>$ Tab. 1).

Auf keine dieser Kategorien würde ein Zusatz von CBD zu einem Lebensmittel zutreffen. Lebensmittelzusatzstoffe haben eine rein technische Funktion in Lebensmitteln, sie können allerdings (unerwünschte) Wirkungen im Organismus haben. CBD wird dagegen ausschließlich zugesetzt, um im Organismus einen Effekt auszulösen. 
-Tab. 1 Funktionsklassen von Lebensmittelzusatzstoffen nach Verordnung 1333 / 2008 / EC.

\begin{tabular}{|l|l|l|}
\hline Süßungsmittel & Füllstoffe & Modifizierte Stärken \\
\hline Farbstoffe & Emulgatoren & Packgase \\
\hline Konservierungsmittel & Schmelzsalze & Treibgase \\
\hline Antioxidationsmittel & Festigungsmittel & Backtriebmittel \\
\hline Trägerstoffe & Geschmacksverstärker & Komplexbildner \\
\hline Säuerungsmittel & Schaummittel & Stabilisatoren \\
\hline Säureregulatoren & Geliermittel & Verdickungsmittel \\
\hline Trennmittel & Überzugs-/Gleitmittel & Mehlbehandlungsmittel \\
\hline Schaumverhüter & Feuchthaltemittel & \\
\hline
\end{tabular}

\section{Schlussfolgerung zur Einstufung als Lebensmittelzusatzstoff}

CBD kann keiner der in der Verordnung 1333 /

2008 / EC definierten Kategorien von Lebensmittelzusatzstoffen zugeordnet werden.

\section{Sind Cannabis und CBD „Novel Food“?}

Unter dem Begriff „neuartiges Lebensmittel“ (Novel Food) versteht man alle Lebensmittel, die vor dem 15. Mai 1997 nicht in nennenswertem Umfang in der Europäischen Union für den menschlichen Verzehr verwendet wurden, und die in mindestens eine der in Artikel 3 der Novel-Food-Verordnung 2015 / 2283 / EC genannten Kategorien fallen, und / oder wenn sie durch ein neuartiges, nicht übliches Verfahren hergestellt werden ( $\triangleright$ Tab. 2).

Ein neuartiges Herstellverfahren wäre auch die Anreicherung von Hanföl oder anderen Speiseölen mit CBD-Konzentraten, isoliertem oder synthetischem CBD. Selbst wenn ein Stoff als solcher (z. B. ein Vitamin) als nicht neuartig anerkannt ist, gilt dies nicht automatisch auch für dessen Zusatz zu einem Nahrungs(ergänzungs)mittel. Dieser Zusatz kann dazu führen, dass ein neuer Antrag auf Zulassung zu stellen ist.

Zugelassene neuartige Lebensmittel sind seit dem 1. Januar 2018 in einer Positivliste, der sogenannten

-Tab. 2 Kategorien von Lebensmitteln, für die NovelFood-Anträge in Betracht zu ziehen sind.

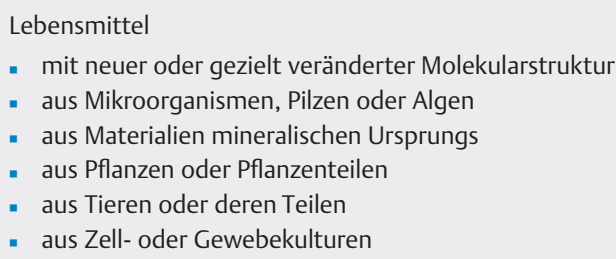

Unionsliste, aufgeführt. Darin aufgelistete Lebensmittel dürfen von allen Lebensmittelherstellern in den Verkehr gebracht werden, wenn die dort angegebenen Verwendungsbedingungen, Kennzeichnungsvorschriften und Spezifikationen eingehalten werden.

CBD wird in dieser Liste eindeutig als eine Substanz genannt, die nicht als herkömmliches Lebensmittel anerkannt werden kann, und für die ein Antrag auf Registrierung als Novel Food gestellt werden muss (https://ec.europa.eu/foo d/safety/novel_food/catalogue/search/public/? event=home\&seqfce $=940$ \&ascii $=$ C\#).

Diese Vorgabe gilt unabhängig von der Herkunft des CBDs (aus natürlichen Quellen oder aus Synthese). Sie gilt auch unabhängig von der Art des Lebensmittels, also auch dann, wenn das CBD anderen Lebensmitteln wie z. B. Hanföl zugesetzt ist, die ihrerseits kein Novel Food wären (https://www.bvl.bund.de/DE/Arbeitsbereiche/01 _Lebensmittel/04_AntragstellerUnternehmen/13_FAQ/F AQ_Hanf_THC_CBD/FAQ_Cannabidiol_node.html).

Novel-Food-Anträge sind lebensmittelspezifisch zu stellen. Ein Antrag für ein CBD-angereichertes Hanföl als Nahrungsmittel würde nicht automatisch auch für einen Mundspray mit CBD oder CBD-angereicherte Gummibärchen gelten, die als Nahrungsergänzungsmittel vermarktet werden. Solche Produktformen würden sich nicht nur in der Formulierung, sondern auch im regulatorischen Status unterscheiden und damit getrennte Anträge erfordern.

Trotz der eindeutigen Kennzeichnung von CBD als registrierungspflichtige Substanz in der Novel-Food-Datenbank der EU wird bezüglich der Verwendung von CBD in Lebensmitteln auf dem Binnenmarkt der EU eher uneinheitlich vorgegangen. CBD wurde in Rumänien für uneingeschränkt verkehrsfähig erklärt, im Widerspruch zum Novel-Food-Register der EU. Cannabis als Pflanze ist auf der sogenannten BELFRIT-Liste für erlaubte 
Nahrungsergänzungsmittel in Belgien, Frankreich und Italien zu finden, ohne eine Dosiseinschränkung bezüglich CBD.

Hanfsamen, Hanföl und Hanfsamenmehl waren schon lange vor dem Stichtag für die Novel-Food-Regelung am 15. Mai 1997 als Lebensmittel im Gebrauch. Sie sind daher nicht neuartig. In der Praxis gelten bei der Betrachtung der Verkehrsfähigkeit von Hanfsamenzubereitungen die anerkannten Grenzwerte für THC. THC in Lebensmitteln aus Hanfsamen resultiert aus einer bei der Samenernte unvermeidbaren Kontaminationen durch Kontakt mit Blüten.

Derzeit sind über 50 Anträge auf Novel Food bei der EU anhängig, viele davon mit synthetischem CBD. Die in diesen Anträgen empfohlenen Tagesdosen sind mit $150 \mathrm{mg} /$ Tag verhältnismäßig hoch (z. B. https://ec.europa.eu/ food/sites/food/files/safety/docs/novel-food_sum_ongo ing-app_2019-0935.pdf), was von den Antragstellern mit dem Fehlen von Toxizität bis zu Dosen von $1200 \mathrm{mg} / \mathrm{Tag}$ und mehr begründet wird. Von diesen Anträgen ist noch keiner beschieden (Stand 19. Januar 2021).

In diesem Zusammenhang ist zu hinterfragen, wie ein Antrag auf Registrierung als Novel Food aus regulatorischer Sicht zu bearbeiten ist. Die Inhalte der Anträge werden durch Artikel 10 der Novel-Food-Direktive 2015 / 2283 / EC geregelt, wobei der Schwerpunkt der Angaben auf der Beschreibung der Zusammensetzung des Lebensmittels, dessen Herstellungsverfahren und dessen toxikologische Eigenschaften liegt. Nur ein Punkt regelt etwas undeutlich die Anforderung für „einen Vorschlag für die Bedingungen der beabsichtigten Verwendung und für spezifische Anforderungen an eine Kennzeichnung, die für die Verbraucher nicht irreführend ist, oder eine nachprüfbare Begründung dafür, weshalb diese Elemente nicht erforderlich sind“. Für die einzureichenden Unterlagen gibt es eine administrative Anleitung der EFSA in Form einer Checkliste, mit deren Hilfe die EFSA die Vollständigkeit des Antrags überprüft [10]. Der größte Teil dieser Liste betrifft rein technische und toxikologische Angaben, die mit der eigentlichen Definition als Lebensmittel nicht im Zusammenhang stehen.

Gefordert wird in dieser Checkliste auch die Darstellung einer Historie der Exposition mit der Substanz. Es muss die Bevölkerungsgruppe beschrieben werden, in der das Lebensmittel Teil der Ernährung war, sowie seine Rolle in der Ernährung. Da dies bei wirklich neuartigen Substanzen nicht möglich ist, ist davon auszugehen, dass diese Beschreibungen lediglich supportiv für die Entscheidung sind. Damit setzen aber Novel-Food-Anträge bereits voraus, dass das zur Genehmigung eingereichte Lebensmittel tatsächlich auch ein Lebensmittel ist.

\section{Schlussfolgerung zur Registrierungspflicht als} Novel Food

CBD ist im EU Novel-Food-Katalog als Substanz aufgenommen, für die ein Antrag auf Registrierung als neuartiges Lebensmittel zu stellen ist. Derzeit sind Produkte mit CBD wie z. B. CBD-Öle als Lebensmittel nicht verkehrsfähig. Da die Anträge auf Novel Food aber eher technischer und toxikologischer Natur sind, wird dadurch nicht beantwortet, ob CBD überhaupt ein Lebensmittel sein kann. Die Beantwortung dieser Frage sollte eigentlich der Betrachtung der Anwendungssicherheit vorgeschaltet sein. Hier besteht Änderungsbedarf an den Checklisten der EFSA.

- Abb. 5 stellt nach dem Verständnis der Autoren eine Entscheidungshilfe dar, ob es sich bei Cannabiszubereitungen um ein Lebensmittel handeln kann. Wendet man konsequent die Definitionen der Gesetze an, ergibt sich keine Situation, derzufolge CBD-Produkte verkehrsfähige Lebensmittel sein könnten.

\section{Ist CBD ein Betäubungsmittel?}

Die rechtlichen Bestimmungen für die nationalen Gesetzgebungen für das Betäubungsmittelrecht basieren auf dem internationalen „Einheitsabkommen über Betäubungsmittel“ der Vereinten Nationen von 1961, das als völkerrechtlicher Vertrag in Deutschland im Jahr 1973 ratifiziert wurde. Dieses Abkommen wurde seither angepasst und enthält Bestimmungen zu Cannabis, die in das deutsche Betäubungsmittelgesetz (BtMG, bezüglich Cannabis geändert am 10.3.2017) übernommen wurden.

Das BtMG führt in Anlage I die Stoffe auf, die als nicht verkehrsfähig betrachtet werden. Explizit aufgeführt sind darin „Pflanzen und Pflanzenteile der zur Gattung Cannabis gehörenden Arten“. Dies bedeutet zunächst, dass Cannabispflanzen und deren Teile grundsätzlich als nicht verkehrsfähige BtM gelten. Für Arzneimittel wurde in Anlage III eine eigene Ausnahme geschaffen, auf die noch zurückzukommen ist. Das BtMG macht hinsichtlich der Einstufung von Cannabispflanzen und Pflanzenteilen eine wesentliche Einschränkung. Das Gesetz befreit diese Pflanzen und Pflanzenteile für die Verwendung außerhalb von Arzneimitteln dann von der Einstufung als BtM, wenn sie entweder von einer im Europäischen Sortenregister erfassten Cannabissorte stammen oder, wie im Sortenregister bereits vordefiniert, der THC-Gehalt maximal $0,2 \%$ beträgt. Diese Sorten werden als Nutzhanf bezeichnet (>Abb. $\mathbf{6})$.

Allerdings wird diese Ausnahme gleich wieder eingeschränkt: Der Handel mit Nutzhanf ist ausschließlich zu gewerblichen oder wissenschaftlichen Zwecken gestattet, 


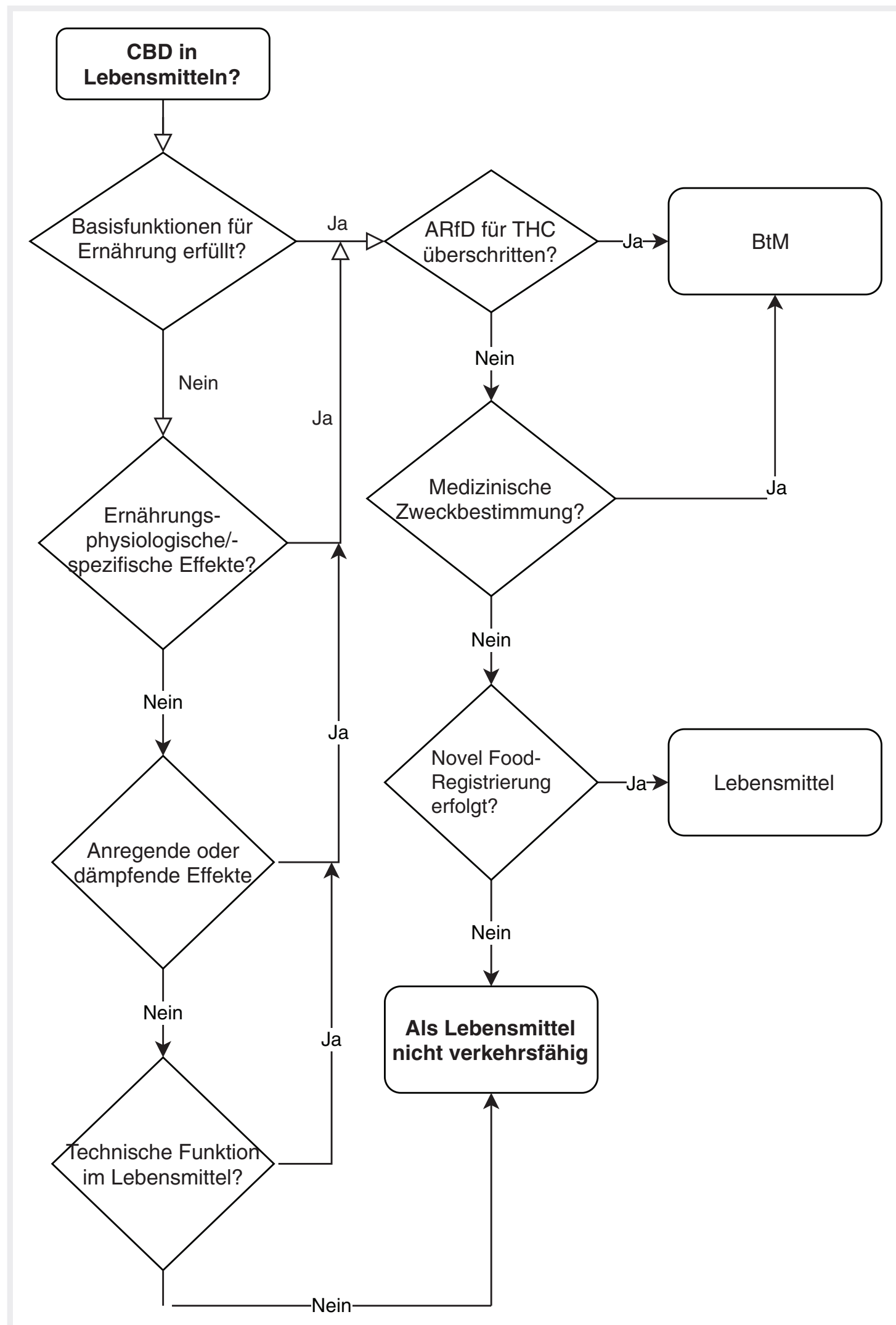

-Abb. 5 Entscheidungshilfe zur Verwendbarkeit von CBD in Lebensmitteln. Die Beantwortung der Hauptfragen führt nach dem Verständnis der Autoren in allen Fällen dazu, dass CBD-Produkte keine verkehrsfähigen Lebensmittel sein können. ARfD = akute Referenzdosis [9] 


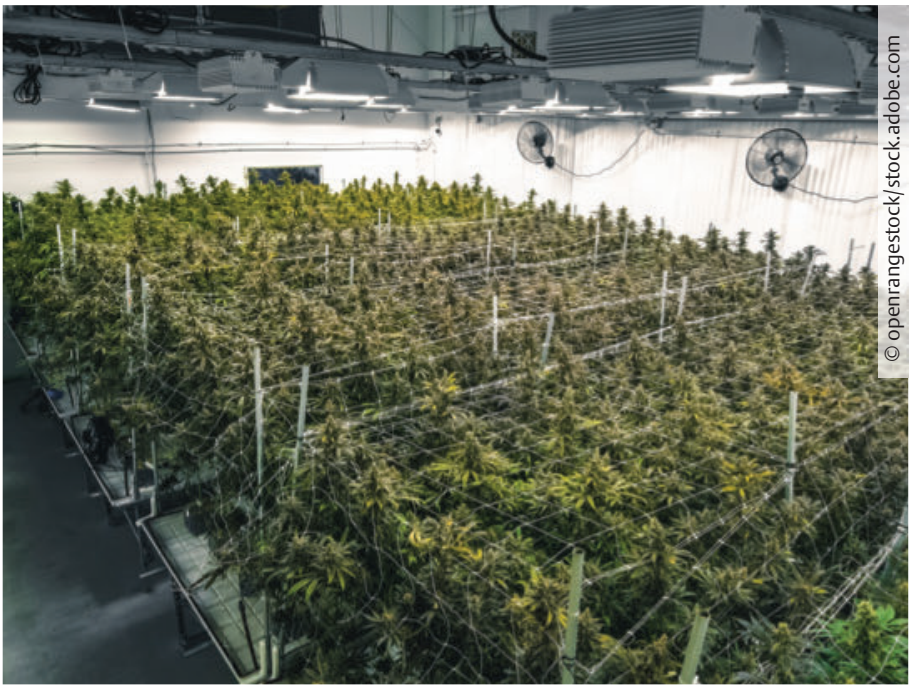

-Abb. 6 Cannabisanbau für medizinische Zwecke darf in Deutschland nur unter speziell gesicherten Bedingungen erfolgen. @ openrangestock / stock.adobe.com

und dann auch nur, wenn ein Missbrauch ausgeschlossen werden kann (https://www.bfarm.de/SharedDocs/FAQs/D E/BtmGrundstoffeAMVV/Cannabis/cannabis-faq14.html). Sinn dieser auf den ersten Blick verwirrenden Regelungen des BtMG war, dass durch die Einschränkung des Handels mit Hanfpflanzenteilen (mit Ausnahme von Samen) sichergestellt werden sollte, dass z. B. textilverarbeitende Betriebe rechtssicher mit Faserhanfsorten arbeiten können. Die Abgabe von Hanfpflanzenmaterial an Endverbraucher war dagegen nie vorgesehen, weil auch bei sehr niedrigen THC-Gehalten ein Missbrauch von Duftkissen mit Hanffüllung, von Tabakersatzprodukten oder Hanftee nicht auszuschließen ist [2].

Hinsichtlich der Formulierung im BtMG ist wesentlich, dass das BtMG von „Pflanzenteilen“, aber nicht von „Pflanzenbestandteilen" spricht. Als Pflanzenbestandteile sind Extrakte und phytochemische Einzelstoffe anzusehen, wenn sie keine eigentlichen Pflanzenteile mehr enthalten. Duftkissen, Hanfzigaretten und Hanftees bestehen also aus Pflanzenteilen, Cannabisextrakte aber nicht [21]. Für die Extrakte würden die Definitionen auf den ersten Blick bedeuten, dass sie, weil vom BtMG nicht erfasst, kein BtM wären. Hier kommt aber der THC-Gehalt ins Spiel: THC ist seinerseits als BtM in Anlage I des BtMG gelistet. Daher können Cannabisextrakte ebenfalls BtM sein, wenn sie THC enthalten. Die entscheidende Menge orientiert sich zunächst an der Vorgabe der 0,2\% THC für Nutzhanf, die sich ihrerseits aber nur auf den Stoff selbst bezieht und die Dosierung und damit die tatsächliche Exposition nicht berücksichtigt. Die in der Praxis von den Überwachungsbehörden angewandten Limits werden im nächsten Abschnitt dieser Analyse dargestellt. Sie bewirken, dass Cannabisextrakte mit CBD aufgrund ihres zwar relativ niedrigen, aber eben nicht vernachlässigbaren Gehaltes an THC relativ schnell als BtM eingestuft werden können.
Eine Ausnahme von der BtM-Einstufung von Cannabis besteht außerdem für Samen und daraus hergestellte Lebensmittel, wie z. B. Hanföl. Sie sind explizit kein BtM. Als Arzneimittel ist Cannabis in Anlage III des BtMG dagegen explizit freigegeben [2]. In diesem Fall muss das Pflanzenmaterial aus staatlich kontrollierten Quellen bezogen werden. In Deutschland ist das die beim BfArM angesiedelte Cannabisagentur.

\section{Zum Begriff „Pflanzenteile“}

CBD ist im BtMG nicht genannt, es ist damit auch kein Betäubungsmittel. CBD wird entweder synthetisch hergestellt oder in Form angereicherter Extrakte aus Cannabisblüten mit -blättern gewonnen. Hier kommt nun eine Diskussion um den Begriff der "Pflanzenteile“ ins Spiel: In Gerichtsurteilen, insbesondere in einem Urteil des Oberlandesgerichtes Hamm vom 21. Juni 2016, wurde angereicherter Cannabisextrakt mit Pflanzenteilen gleichgesetzt. Damit kam das Gericht zu der Schlussfolgerung, dass grundsätzlich alle Cannabisprodukte mit Ausnahme von Samen und Samenöl unter die Klassifizierung als Betäubungsmittel fallen und nicht an Endverbraucher abgegeben werden können - und dies unabhängig vom THC-Gehalt [6]! Dieser Argumentation folgt auch das Umweltministerium des Landes Nordrhein-Westfalen in einer Stellungnahme [2]. Erst nach Verarbeitung zu einem Produkt, das nicht mehr zu Rauschzwecken eingesetzt werden kann, wäre die Abgabe an den Endverbraucher nach Ansicht des Oberlandesgerichtes Hamm wieder möglich. Ein Beispiel wäre Hanfbier, zu dessen Herstellung Cannabisblüten mit weniger als 0,2\% THC eingesetzt werden können. Das Bier kann an die Endverbraucher abgegeben werden, die Blüten selbst dagegen nicht. Damit können Hanfblüten wie z. B. in Hanftee aber auch kein Nahrungsmittel sein. Der Wissenschaftliche Dienst des Deutschen Bundestags hat hier durch die Unterscheidung zwischen Pflanzenteilen und Pflanzenbestandteilen etwas schärfer differenziert [21]. Demnach müssten Cannabisprodukte zumindest noch Teile der Cannabispflanze in „substanzieller Form“ enthalten, wie es bei Hanfblütentees oder Duftkissen der Fall ist. CBD-Öle enthalten aber keine Pflanzenteile, sondern nur noch Pflanzenbestandteile, weshalb sie nur dann unter Betäubungsmittelrecht fallen würden, wenn sie auch THC enthalten [21].

Der Wissenschaftliche Dienst stellt allerdings nicht fest, ab welcher THC-Menge der Extrakt BtM wäre. Der reine Nachweis von THC sollte dazu nicht ausreichen, weil auch Hanfsamen aufgrund unvermeidlicher Kontaminationen zwar geringe, aber analytisch nachweisbare Mengen an THC enthalten. Hier kommen in der Praxis die unten dargestellten Grenzwerte zum Tragen.

\section{Synthetisches CBD}

Diskrepanzen in der aktuellen Anwendung der Rechtslage sind insbesondere bei der Verwendung von synthetischem CBD zu erkennen: Da CBD als Reinstoff kein 
Betäubungsmittel ist und bei synthetischem CBD die Einschränkungen des BtMG hinsichtlich Cannabis sativa und dessen Pflanzenteilen nicht zutreffen, käme hier eine Einstufung als BtM nicht infrage. Entsprechend wurde CBD rechtlich unterschiedlich behandelt, je nachdem, ob es chemischen Ursprungs oder aus der Pflanze isoliert war. Hierzu hat das EuGH am 19. November 2020 ein richtungsweisendes Urteil gefällt (C663-18). CBD ist generell kein BtM, weil der Substanz die psychoaktiven Effekte fehlen, die eine Einstufung als BtM erst ermöglichen. Eine Unterscheidung zwischen synthetischem und aus Cannabis isoliertem CBD ist daher nicht gerechtfertigt. Die Verkehrsfähigkeit von CBD als Reinstoff darf also nicht eingeschränkt werden, nur weil die Quelle pflanzlich ist. Die Behörden müssen sich bei Einschränkungen des Warenverkehrs grundsätzlich auf darstellbare Risiken berufen, rein hypothetische Risiken reichen für ein Verbot CBD-haltiger Produkte nicht aus.

Allerdings wäre hier zu berücksichtigen, dass auch synthetisches CBD aufgrund von Syntheseverunreinigungen THC enthalten kann - grundsätzlich können die gleichen Expositionsargumente hinsichtlich THC sowohl auf synthetisches CBD als auch auf CBD aus
Cannabis angewandt werden. Somit kann auch synthetisches CBD bei entsprechender Dosierung zum BtM werden.

Das EuGH-Urteil scheint zunächst mehr Rechtssicherheit für CBD-haltige Produkte wie CBD-Öle geschaffen zu haben, was auch in aktuellen Updates behördlicher Stellungnahmen umgesetzt wurde [17]. Unverständlich war aber, dass aktuell die EU-Kommission aus dem EuGH-Urteil herauslas, dass nun CBD-Produkte als Lebensmittel eingestuft werden können (https://ec.europa.eu/ger many/news/20201203-cannabidiol-produkte_de). Tatsächlich hat das EuGH mit keinem Wort die Aussage getroffen, CBD sei Lebensmittel - der zugrunde liegende Rechtsstreit befasste sich auch gar nicht mit dieser Frage. Schlimmer noch: Die Einschätzung der EU-Kommission würde zu einer weiteren Ungleichbehandlung führen, denn nach der Einordnung der Bundesopiumstelle sind Arzneimittel, die CBD-basierte Zubereitungen aus Medizinalcannabis enthalten, automatisch BtM, auch wenn sie weniger THC enthalten, als in einem Lebensmittel auf der Basis der Referenzwerte enthalten sein darf (https://www.bfarm.de/SharedDocs/FAQs/DE/BtmG rundstoffeAMVV/Cannabis/cannabis-faq14.html)! 


\section{Schlussfolgerung zur Einstufung von CBD als Betäubungsmittel}

CBD als Reinsubstanz ist eindeutig nicht BtM und fällt daher als solches nicht unter die Regelungen des BtMG. Dies bedeutet aber nicht im Umkehrschluss, dass CBD nun ein Lebensmittel ist. Zudem können CBD-haltige Lebensmittel allein durch ihren Gehalt geringer Mengen an THC bei entsprechender Verzehrsempfehlung die Grenze zum BtM überschreiten. Arzneimittel mit Cannabisextrakten als Wirkstoff gelten generell als BtM.

\section{Definition von Dosisgrenzwerten}

Grenzwerte sind wichtig für die Frage, ab wann eine Zubereitung nicht mehr als sicheres Lebensmittel im Sinne der EU-Verordnung 178/2002 gelten kann. Grenzwerte existieren für THC, nicht aber für CBD. Limits für THC in Lebensmitteln wurden bereits im Jahr 2000 vom Bundesinstitut für Risikobewertung (BfR) vorgeschlagen: Demnach dürfen alkoholische und nichtalkoholische Getränke auf Hanfbasis nicht mehr als $5 \mu \mathrm{g} / \mathrm{kg}$ THC enthalten, Speiseöle (Hanföl) nicht mehr als $5000 \mu \mathrm{g} / \mathrm{kg}$ und andere Lebensmittel nicht mehr als $150 \mu \mathrm{g} / \mathrm{kg}$ [4]. Wie bereits erwähnt, fallen diese Lebensmittel auf der Basis von Hanfsamen nicht unter das Betäubungsmittelrecht und sind auch keine neuartigen Lebensmittel.

Im Jahr 2015 schlug die EFSA für THC einen „Lowest Observed Adverse Effect Level“ (LOAEL) von $2,5 \mathrm{mg}$ THC/ Tag vor und errechnete daraus eine als sicher betrachtete Exposition in Form einer „akuten Referenzdosis“ ARfD von täglich $1 \mu \mathrm{g} / \mathrm{kg}$ Körpergewicht [9]. Dieser Referenzwert dient noch immer als Basis für Bewertungen, insbesondere weil vom BfR und den Landesuntersuchungsämtern regelmäßig von zum Teil massiven Überschreitungen dieser Grenzwerte berichtet wird [1],[5], [11],[15].

Die aktuelle Entscheidungsgrundlage des Umweltministeriums des Landes NRW für die Einstufung von Produkten auf Cannabisbasis ist vergleichsweise einfach: Wenn die akute Referenzdosis von $1 \mu \mathrm{g} / \mathrm{kg}$ Körpergewicht THC überschritten wird oder wenn allgemein eine Zufuhr von 2,5 mg THC / Tag erreicht wird, gelten hanfhaltige Nahrungsmittel nicht mehr als sicher und damit nicht als verkehrsfähig [2].

\section{PRAXISBEISPIEL}

Ein CBD-Öl mit einem ausgelobten Gehalt von $20 \%$ CBD und 0,1\% THC würde einer Menge von $200 \mathrm{mg} /$ $\mathrm{ml} \mathrm{CBD}$ und $1 \mathrm{mg} / \mathrm{ml}$ THC entsprechen. Die Menge an THC in der Zubereitung wäre also erst einmal unterhalb des Schwellenwerts von 0,2\% nach
Betäubungsmittelrecht. Auf den ersten Blick deutet dies nicht unmittelbar auf ein Problem hin. Allerdings ist auch die Einnahmeempfehlung einzubeziehen. Eine typische Dosis wäre 0,5 ml/Tag, entsprechend $0,5 \mathrm{mg}$ THC. Die Kalkulationen der Behörden gehen von einem Körpergewicht von $70 \mathrm{~kg}$ aus. Auf der Basis des Grenzwertes von 1 mg/kg Körpergewicht darf die Tagesdosis eines Cannabis-Produktes die Menge von $70 \mu \mathrm{g}$ THC nicht überschreiten. Bei einer Tagesdosis von $0,5 \mathrm{mg}=$ $500 \mu \mathrm{g}$ wird der Grenzwert aber bereits um das Siebenfache überschritten. Die Untersuchungsbehörde würde diesen Fall an die Strafverfolgungsbehörden weitergeben [2].

CBD gilt auch in hohen Dosen als sehr gut verträglich. Die CBD-Gehalte der vermarkteten Produkte sind extrem heterogen und belegen in der Regel eine Anreicherung in den jeweiligen Produkten. Zur Orientierung: Laut Monitoringbericht des Bundesamtes für Verbraucherschutz und Lebensmittelsicherheit (BVL) aus dem Jahr 2019 enthalten Hanföle im Median 23 mg CBD / kg Öl [7]. Da Hanföl von Natur aus kein CBD enthält, können die sehr hohen Gehalte von CBD in sogenannten CBD-Ölen nur durch gezielten Zusatz von CBD aus Blütenextraktkonzentraten oder von synthetischem CBD erreicht werden. CBD-Öle sind daher keine natürlichen Lebensmittel.

Unklar ist bislang, ab welcher Menge von CBD von pharmakologischen Effekten auszugehen ist. Für einen pharmakologischen Effekt für medizinische Zwecke werden typischerweise CBD-Dosen von mindestens $1000 \mathrm{mg} /$ Tag benötigt [8]. Die orale Bioverfügbarkeit von CBD ist schlecht, sie beträgt nur ca. 6\% [12]. Moderne galenische Formulierungen (z. B. mittels Lipiden und Mikronisierung) verbessern die Bioverfügbarkeit erheblich und ermöglichen damit pharmakologische Effekte auch bei deutlich geringeren Dosen [13]. Dies könnte ein Grund dafür sein, dass sich das BfArM in einer Stellungnahme für eine pauschale Grenzdosis von $18 \mathrm{mg}$ aussprach [12].

\section{Schlussfolgerung zu Grenzwerten}

Cannabisextrakte in Lebensmitteln, die neben CBD auch geringe Mengen an THC enthalten, überschreiten aufgrund ihrer Dosierungsempfehlung vielfach die vom BfR definierten Tageshöchstmengen und können damit die Grenze zum BtM überschreiten. Für CBD existiert aktuell keine Grenzdosis. Eine solche wäre aber nur dann erforderlich, wenn mit CBD angereicherte Produkte die Definition eines Lebensmittels erfüllen würden. Da dies nicht der Fall ist, ist eine Festlegung nicht unbedingt erforderlich. 


\section{CBD in Arzneimitteln}

Arzneimittel im Sinne des Arzneimittelgesetzes (AMG) und der europäischen Direktive 2001 / 83 / EC sind nach der Definition in §2 AMG „Stoffe oder Zubereitungen aus Stoffen, die

1. zur Anwendung im oder am menschlichen oder tierischen Körper bestimmt sind und als Mittel mit Eigenschaften zur Heilung oder Linderung oder zur Verhütung menschlicher oder tierischer Krankheiten oder krankhafter Beschwerden bestimmt sind oder

2. die im oder am menschlichen oder tierischen Körper angewendet oder einem Menschen oder einem Tier verabreicht werden können, um entweder

a. die physiologischen Funktionen durch eine pharmakologische, immunologische oder metabolische Wirkung wiederherzustellen, zu korrigieren oder zu beeinflussen oder

b. eine medizinische Diagnose zu erstellen.

Das AMG stellt zwar in § 2(3) Nr. 1 fest, dass Lebensmittel zunächst keine Arzneimittel sind, schränkt dies aber durch die Zweifelsfallregelung in Absatz 3a wieder ein:

„Arzneimittel sind auch Erzeugnisse, die Stoffe oder Zubereitungen aus Stoffen sind oder enthalten, die unter Berücksichtigung aller Eigenschaften des Erzeugnisses unter eine Begriffsbestimmung des Absatzes 1 fallen und zugleich unter die Begriffsbestimmung eines Erzeugnisses nach Absatz 3 fallen können.“

Das bedeutet, dass auch Stoffe, die als Lebensmittel, aber auch als Kosmetika oder Tabakerzeugnisse vertrieben werden, aufgrund ihrer Eigenschaften zum Arzneimittel werden können. Durch den Sachverständigenausschuss für Verschreibungspflicht wurde CBD 2016 eindeutig als pharmakologisch aktive Substanz eingestuft und damit die Verschreibungspflicht begründet [3] ( $>$ Info).

INFO

CBD-Effekte an Enzymen und Rezeptoren (laut BfArM) [3]

- CBD ist ein Antagonist am G-Protein gekoppelten Cannabinoidrezeptor GPR55

- CBD hemmt den zellulären lonenkanal TRMP8, den $\alpha 1$-Adrenozeptor und den $\mu$-Opioidrezeptor

- CBD hemmt die Fettsäureamidhydrolase, was den Abbau des endogenen Cannabinoids Anandamid verhindert

- CBD blockiert (nicht näher genannte) Calciumkanäle

- CBD hemmt die Wiederaufnahme von Noradrenalin, Dopamin, Serotonin und $\mathrm{\gamma}$-Aminobuttersäure

- CBD bindet an 5-HT 1 -Rezeptoren
Cannabiszubereitungen, THC und CBD sind in Form von Arzneimitteln mit definierter Dosis und einer definierten Indikation zur Behandlung von Erkrankungen zugelassen. Sie sind auch als Rezeptursubstanz erhältlich. Für CBD gibt es eigene Monografien in DAC und NRF. Einsatz findet CBD als Arzneimittel vor allem bei Erkrankungen mit spastischen Symptomen, wie zum Beispiel der Multiplen Sklerose oder bei Epilepsie. Diskutiert wird aber auch der Einsatz unter anderem bei Angststörungen, Nervenentzündungen, neurodegenerativen Erkrankungen und bestimmten Krebsformen. Die pharmakologischen Effekte und die möglichen Einsatzgebiete sind in umfangreichen Monografien dargestellt [14],[18].

Für Arzneimittel mit dem Wirkstoff CBD gibt es zwei grundsätzliche Wirkstoffkategorien:

- Produkte mit CBD-Reinstoffen (Reinheitsgrad > $98 \%$ nach der DAC-Monografie)

- CBD-haltige Cannabisextrakte

In beiden Fällen gilt, dass CBD auf der Liste der verschreibungspflichtigen Arzneistoffe der Arzneimittelverschreibungsverordnung (AMVV) gelistet ist. CBD in Arzneimitteln untersteht grundsätzlich dem Betäubungsmittelrecht, wenn es in Form eines Cannabisextraktes vorliegt und eine medizinische Zweckbestimmung hat, unabhängig vom tatsächlichen THC-Gehalt (https://www. bfarm.de/SharedDocs/FAQs/DE/BtmGrundstoffeAMVV/Ca nnabis/cannabis-faq14.html). Diese behördliche Regelung steht im Widerspruch zum aktuellen EuGH-Urteil.

Wie bereits dargestellt, überschreiten aber CBD-haltige Lebensmittel durch ihre Tagesdosis von THC sehr häufig die Grenze zur Einstufung als BtM. In unzähligen Artikeln im Internet wird dem Verbraucher suggeriert, dass CBD bei verschiedenen Erkrankungen wirksam sei. Da ein ernährungsphysiologischer Nutzen nicht bekannt ist, muss davon ausgegangen werden, dass der Verbraucher CBDProdukte in erster Linie aus gesundheitlichen Gründen anwendet, also mit einer medizinischen Zweckbestimmung. Auch wenn diese Zweckbestimmung nicht explizit auf dem Etikett des CBD-Produktes erwähnt wird, so ist sie aufgrund vielfältiger Artikel im Bewusstsein des Verbrauchers präsent. Damit ist es für die Hersteller von CBD-Produkten, die als Nahrungs(ergänzungs)mittel, Kosmetika, Aromata oder Tabakersatzprodukte vertrieben werden, nicht mehr erforderlich, selbst eine Indikation auf der Produktkennzeichnung auszuloben-dies würde ohnehin an den Hürden des Werberechts scheitern. Es stellt sich also die Frage, welchem Zweck eine Anreicherung von CBD in Nahrungsmitteln oder anderen Verbraucherprodukten dienen sollte, wenn nicht der Therapie von Beschwerden, also mit einem arzneilichen Zweck. Lebensmittel können zwar vielleicht zur Gesunderhaltung beitragen, sie können aber nicht zur Therapie von Beschwerden verwendet werden. Damit wäre die Schwelle zum Arzneimittel überschritten. Daran werden 
sich CBD-Zubereitungen hinsichtlich der Health Claims in der Bewertung durch die EFSA messen lassen müssen.

\section{Schlussfolgerung}

CBD-Zubereitungen mit medizinischer Zweckbestimmung sind verschreibungspflichtige Arzneimittel. Sie haben eindeutige pharmakologische Eigenschaften. Cannabisextrakte werden darüber hinaus unabhängig vom THC-Gehalt grundsätzlich als BtM eingestuft. Die Zweifelsfallregelung nach § 2

(3a) AMG zur Einstufung anderer Produktformen als Arzneimittel ist auf CBD-Produkte anwendbar, unabhängig davon, ob ihnen eine konkrete medizinische Zweckbestimmung zugeschrieben wird.

\section{Darf CBD in Lebensmitteln mit Gesundheitsaussagen beworben werden?}

Gesundheitliche Aussagen auf Lebensmitteln unterliegen einer Prüfung und Genehmigung nach den Vorgaben der „Health Claim Direktive“ 1924 / 2006/ EC. Es muss dazu bei der EFSA ein Health Claim-Antrag gestellt und genehmigt werden. Die Health Claims sind entweder allgemeine Aussagen im Sinne einer Gesunderhaltung einer Körperfunktion, oder sie können die Reduktion eines Risikos für eine bestimmte Erkrankung betreffen. In beiden Fällen richten sich die Produkte an die gesunde Bevölkerung. Die Grenzen der Aussagen werden von der EFSA sehr eng gefasst. Wenn sich die Aussage auf die Zufuhr einer bestimmten Substanz bezieht, müssen auch die zugeführten Mengen und ihr Zusammenhang mit einer gesunden Körperfunktion anhand wissenschaftlicher Daten dargestellt werden. Die Menge muss für den Lebensmittelzweck angemessen sein. Es ist in Health Claim-Anträgen grundsätzlich nicht möglich, sich auf Studien mit einer arzneilichen Anwendung zu beziehen.

Bislang gibt es für CBD-haltige Lebensmittel keinen Claim. Es ist ja noch nicht einmal die Frage des Novel-Food-Status beschieden, geschweige denn ein ernährungsphysiologischer Nutzen von angereichertem CBD zur Gesunderhaltung des Organismus dargestellt. Den Novel-Food-Status vorausgesetzt, könnte die aktuelle Regelung allerdings die Einführung von CBD durch die Hintertür ohne eigenen Claim erlauben, nämlich dann, wenn sich der Health Claim auf Zusätze von anderen Nährstoffen bezieht, für welche ein Claim bereits genehmigt ist. Allerdings kann es bereits aus der gesetzlichen Definition der Health Claims heraus keinen einzigen Claim geben, der die sehr dezidierten Heilsversprechen CBD-haltiger Nahrungsergänzungsmittel, wie sie im Internet und in Publikumszeitschriften unschwer zu finden sind, abdecken würde: Die WHO kritisiert in ihrem Review aus dem Jahr 2018 die Auslobung von CBD in Nahrungsmitteln bei Epilepsie, Krebs, HIV, Angstbeschwerden, Arthritis, Schmerzen und bei posttraumatischen Belastungsbeschwerden [12].

Solche Indikationsbehauptungen finden sich auch aktuell in der Werbung für CBD-haltige Nichtarzneimittel. Meist gibt es im Umfeld dann einen Disclaimer, der darauf hinweist, dass die genannten Verwendungen und Dosisempfehlungen für den arzneilichen Einsatz gelten und daher die Verwendung auf eigene Gefahr erfolgt, sollte jemand auf die Idee kommen, aus den Informationen eine Anwendungsempfehlung herauszulesen [6]. Auch durch diese Art der Darstellung könnte die Schwelle zum Arzneimittel überschritten und zudem ein Verstoß gegen die Verschreibungspflicht, bei Überschreitung der Tagesdosen für THC ggf. sogar die BtM-Pflicht gegeben sein. Beide Argumente werden von Behörden in der Verfolgung von entsprechenden Verstößen benutzt.

\section{Schlussfolgerung zu gesundheitsbezogenen Aus- sagen für CBD \\ Da die Werbeaussagen arzneiliche Anwendungen betreffen, gleichzeitig aber eine gesundheitsbezo- gene Aussage für subpharmakologische Mengen an CBD fehlt (was den Nachweis einer Grenzdosis ohne pharmakologische Effekte voraussetzen würde), soll- ten gesundheitliche Aussagen zu CBD außerhalb des Arzneimittelbereichs nicht möglich sein.}

\section{Fazit}

CBD ist nach allen in dieser Darstellung diskutierten Kriterien unabhängig von der Herkunft aus Synthese oder Anreicherung ein eindeutiger, verschreibungspflichtiger Arzneistoff, der bei Herkunft aus Cannabisextrakten zudem unter das BtMG fällt und somit nach den aktuellen Regeln kein Lebensmittel sein kann:

- CBD erfüllt weder die Anforderungen der Funktionen eines Lebensmittels noch wurde ein ernährungsphysiologischer Nutzen beschrieben.

- CBD fällt in keine Kategorie für Lebensmittelzusatzstoffe.

- CBD-Extrakte oder -Konzentrate überschreiten vielfach die für Lebensmittel definierten Tageshöchstmengen an THC und können dann als BtM eingestuft werden.

- CBD ist aktuell als Stoff klassifiziert, für den ein NovelFood-Antrag zu stellen ist. In Ermangelung eines beschiedenen Novel-Food-Antrags sind Lebensmittel mit CBD wie CBD-Öle aktuell nicht verkehrsfähig. Die Einstufung als Stoff, für den ein Antrag auf Novel Food gestellt werden muss, bescheinigt aber nicht die Eigenschaft als Lebensmittel.

- CBD-Öle werden unter missbräuchlicher Verwendung des Lebensmittelstatus im Sinne von Funktions- und Präsentationsarzneimitteln mit medizinischen Claims 
und pharmakologisch relevanten Dosierungen beworben.

Nach Lebensmittelrecht kann ein Stoff, der als BtM klassifiziert ist, nicht Bestandteil von Nahrungsmitteln sein. CBD selbst ist zwar kein BtM, Cannabisextrakte in Arzneimitteln werden aber automatisch als BtM eingestuft, unabhängig von ihrem THC-Gehalt. Es wäre nicht nachvollziehbar, wenn vergleichbare Zubereitungen als Arzneimittel unter Berufung auf das BtMG den Status eines BtM haben, in Form eines Lebensmittels aber auf den Markt kommen dürften, wobei dann der THC-Gehalt sogar höher sein kann als im Arzneimittel.

Aus den dargestellten Zusammenhängen ergibt sich ein regulatorischer Änderungsvorschlag, der ohne Eingriff in die existierenden Gesetze umsetzbar wäre: Die Checkliste der EFSA zur Vollständigkeit der Unterlagen eines Antrags auf Novel Food [10] müsste lediglich um die Forderung ergänzt werden, die Lebensmitteleigenschaften einer neu zu bewertenden Zubereitung vor dem Hintergrund des Lebensmittelrechtes zu begründen.

Aus den dargestellten Zusammenhängen ergibt sich die offensichtliche Antwort auf die eingangs gestellte Frage: Entgegen der Aussage der EU-Kommission vom 3.12.2020 hat der Wortlaut des EuGH-Urteils vom 19. November 2020 keineswegs eine prinzipielle Einstufung von CBD als Lebensmittel ermöglicht, denn das Gericht hat sich mit dieser Frage gar nicht befasst. Das EuGH-Urteil ändert nichts an den Tatsachen, dass CBD ein verschreibungspflichtiger Arzneistoff ist und dass CBD-haltige Nahrungs(ergänzungs)mittel die Definition eines Lebensmittels nicht erfüllen. Die Kommentierung durch die EUKommission hat leider das Potenzial, erheblich zur Verwirrung beizutragen. Der Handlungsbedarf ist wegen der Irreführung des Verbrauchers zudem dringlich.

\section{ABSTRACT}

Is cannabidiol food or medicine? A positioning

Products containing cannabidiol (CBD) such as CBD oil are marketed in the form of food or dietary supplements. They are often advertised with medical indications for serious illnesses. Applications for Novel Food Status are currently processed at European level. On closer examination, however, the basis for classifying CBD as a foodstuff is questionable. CBD is not only clearly a prescription drug with known pharmacological effects, derived from cannabis extracts, it even has a narcotic status. On the other hand, CBD lacks the food-typical properties to be used in / as food. Even if CBD seems to be toxicologically harmless even in high doses, this does not imply that a drug qualifies to be categorized as novel food just because it has been proven to be safe for consumers.
Interessenkonflikt

K.K. erklärt, dass kein Interessenkonflikt besteht. M.S. arbeitet als sachkundige Person für ein pharmazeutisches Unternehmen, das THC-haltige Cannabisextrakte als Arzneimittel unter BtM-Recht herstellt.

\section{Korrespondenzadresse}

\section{Prof. Dr. med. habil. Karin Kraft}

Lehrstuhl für Naturheilkunde

Zentrum für Innere Medizin

Universitätsmedizin Rostock

Ernst-Heydemann-Str. 6

18057 Rostock, Deutschland

E-Mail: Karin.kraft@med.uni-rostock.de

Die Autorin ist Stellvertretende Vorsitzende der Kommission E des BfArM und Präsidentin der Gesellschaft für Phytotherapie e. V.

\author{
Dr. rer. nat. Mathias Schmidt \\ Herbresearch Germany \\ Wartbergweg 15 \\ 86874 Mattsies, Deutschland \\ E-Mail: schmidt@herbresearch.de \\ Der Autor ist Mitglied des wissenschaftlichen Komitees der \\ ESCOP und Vorsitzender des wissenschaftlichen Kuratoriums \\ der Gesellschaft für Phytotherapie e. V.
}

\section{Literatur}

[1] Jahresbericht 2019. Überwachung, Lebensmittel, Bedarfsgegenstände, Kosmetika, Trinkwasser Futtermittel. Baden-Württemberg: Ministerium für Ländlichen Raum und Verbraucherschutz; 2019. Im Internet: https://mlr.baden-wu erttemberg.de/de/unser-service/publikation/did/jahresbe richt-2019-ueberwachung-lebensmittel-bedarfsgegensta ende-kosmetika-trinkwasser-und-futter/

[2] Hanf, THC, CBD \& Co. Fragen und Antworten zur Beurteilung von Produkten im Rahmen der amtlichen Lebensmittelüberwachung. Land Nordrhein-Westfalen: Ministerium für Umwelt, Landwirtschaft, Natur- und Verbraucherschutz; 2020. Im Internet: https://www.umwelt.nrw.de/fileadmin/redak tion/PDFs/verbraucherschutz/faq_hanf_thc_co.pdf

[3] BfArM. Cannabidiol. Sachverständigen-Ausschuss für Verschreibungspflicht 2016; 19.1.2016. Im Internet: https://ww w.bfarm.de/SharedDocs/Downloads/DE/Arzneimittel/Pharma kovigilanz/Gremien/Verschreibungspflicht/75Sitzung/an lage2.pdf?_blob=publicationFile\& $=1$

[4] BfR. BgVV empfiehlt Richtwerte für THC (Tetrahydrocannabinol) in hanfhaltigen Lebensmitteln. Presseinformation. 2000. Im Internet: https://www.bfr.bund.de/de/presseinformation/20 00/07/bgvv_empfiehlt_richtwerte_fuer_thc_tetrahydrocanna binol_in_hanfhaltigen_lebensmitteln-884.html

[5] BfR. Tetrahydrocannabinolgehalte sind in vielen hanfhaltigen Lebensmitteln zu hoch - gesundheitliche Beeinträchtigungen sind möglich. Stellungnahme Nr. 034/2018. 2018. doi: 10.17590/20181108-075209-0

[6] Blasius H. Harmloser Hanf? Aufgepasst bei Cannabidiol-Produkten in der Apotheke. Dtsch Apotheker Ztg 2018; 158 (50): 52-56. Im Internet: https://www.deutsche-apotheker-zei tung.de/daz-az/2018/daz-50-2018/harmloser-hanf 
[7] Bundesamt für Verbraucherschutz und Lebensmittelsicherheit. Monitoring 2019. BVL-Report 15.3. Berichte zur Lebensmittelsicherheit. Berlin: BVL; 2019. Im Internet: https://www. bvl.bund.de/SharedDocs/Berichte/01_LM_Monitoring/2019_I m_monitoring_bericht.pdf?_blob=publicationFile \& $=4$

[8] Davies C, Bhattacharyya S. Cannabidiol as a potential treatment for psychosis. Ther Adv Psychopharmacol 2019; 9: 2045125319881916. doi:10.1177/2045125319881916

[9] EFSA. Scientific Opinion on the risks for human health related to the presence of tetrahydrocannabinol (THC) in milk and other food of animal origin. EFSA J 2015; 13: 4141. doi:10.2903/j.efsa.2015.4141

[10] EFSA. Administrative guidance on the submission of applications for authorisations of a novel food pursuant to Article 10 of Regulation (EU) 2015/2283. EFSA Supporting Publication 2018: EB-1381. doi:10.2903/sp.efsa.2018.EN-1381

[11] EFSA. Acute human exposure assessment to tetrahydrocannabinol (delta9-THC). EFSA J 2019; 18: 5953. doi:10.2903/j. efsa.2020.5953

[12] WHO. Expert Committee on Drug Dependence. Cannabidiol (CBD). Critical Review Report. Fortieth Meeting, 4-7 June 2018. Geneva: WHO; 2018. https://www.who.int/medicines/access/ controlled-substances/CannabidiolCriticalReview.pdf

[13] Grotenhermen F. Pharmakokinetik der Cannabinoide. In: Müller-Vahl KR, Grotenhermen F, Hrsg. Cannabis und Cannabinoide in der Medizin. Berlin: Medizinisch-Wissenschaftliche Verlagsgesellschaft; 2020: 75-83

[14] Grotenhermen F, Russo E. Cannabis and Cannabinoids. Pharmacology, Toxicology, and Therapeutic Potential. New York: Routledge; 2002

[15] Habel S, Lachenmeier D. Cannabidiol (CBD) - ein Hype mit Gesundheitsrisiko. Karlsruhe: Chemisches und Veterinäruntersuchungsamt; 2019. https://www.ua-bw.de/pub/beitrag.as p?subid=2\&Thema_ID=2\&ID=3021\&Pdf=No\&lang=DE

[16] Hässig A, Liang WX, Schwabl $\mathrm{H}$ et al. Flavonoids and tannins: plant-based antioxidants with vitamin character. Med Hypotheses 1999; 52: 479-481. doi:10.1054/mehy.1997.0686
[17] Lachenmeier D. Update zur Verkehrsfähigkeit von Cannabidiol (CBD)-Produkten. Karlsruhe: Chemisches und Veterinäruntersuchungsamt; 2020. https://www.ua-bw.de/pub/beitrag.as p? subid=2\&Thema_ID=2\&ID=3294\&lang=DE\&Pdf=No

[18] Müller-Vahl K, Grotenhermen F. Cannabis und Cannabinoide in der Medizin. Berlin: Medizinisch-Wissenschaftliche Verlagsgesellschaft; 2020

[19] Nagy DU, Cianfaglione K, Maggi F et al. Chemical characterization of leaves, male and female flowers from spontaneous cannabis (Cannabis sativa L.) growing in Hungary. Chem Biodivers 2019; 16: e1800562. doi:10.1002/cbdv.201800562

[20] Wissenschaftliche Dienste. Verkehrsfähigkeit von Cannabidiol (CBD)-haltigen Lebensmitteln (22. Juli 2019). Berlin: Deutscher Bundestag; 2019. Im Internet: https://www.bundestag. de/resource/blob/655138/c9a79d6ee7414e9c0eb0 d3e63d64236d/WD-5-065-19-pdf-data.pdf

[21] Wissenschaftliche Dienste. Verkehrsfähigkeit von Cannabidiol (CBD)-haltigen nicht medizinischen Produkten in Deutschland (2.Oktober 2020). Berlin: Deutscher Bundestag; 2020. Im Internet: https://www.bundestag.de/resource/blob/804876/a c332e279eafe1a5145e69c6e170976a/WD-5-088-20-pdf-dat a.pdf

[22] Yoon JH, Baek SJ. Molecular targets of dietary polyphenols with anti-inflammatory properties. Yonsei Med J 2005; 46: 585-596. doi:10.3349/ymj.2005.46.5.585

Bibliografie

Zeitschrift für Phytotherapie 2021; 42: 82-94

DOI 10.1055/a-1336-6273

ISSN 0722-348X

(c) 2021. Thieme. All rights reserved.

Georg Thieme Verlag KG, Rüdigerstraße 14, 70469 Stuttgart, Germany 